\title{
Remote linear radio frequency PECVD deposited high quality a-Si:H(p) layers and their application in Si heterojunction structures
}

\author{
Y. Wu, C. Devilee, B. B. Van Aken, K. Boulif, W. J. Soppe, A. W. Weeber, L. J. Geerligs \\ ECN Solar Energy, PO Box 1, 1755 ZG Petten, The Netherlands
}

\begin{abstract}
In this paper, we report on deposition and properties of high quality boron doped p-type amorphous $\mathrm{Si}(\mathrm{a}-\mathrm{Si}: \mathrm{H}(\mathrm{p}))$ layers on n-type float zone $\mathrm{Si}(100)$ wafers by remote linear radio frequency plasma-enhanced CVD. The a-Si:H(p) layers show excellent surface passivation that is comparable to the one of a-Si:H intrinsic layers (a$\mathrm{Si}: \mathrm{H}(\mathrm{i}))$, and high stability of the passivation when stored in the dark. Additionally, the measured dark conductivity of deposited a-Si(p) is increased up to $>7 \times 10^{-6} \mathrm{~S} / \mathrm{cm}$ by annealing. In a Si heterojunction cell structure, the a-Si:H(p) layer will be the emitter on an n-type base wafer. The effective lifetime of test structures of a-Si(p)/c-Si(n)/a-Si(n) has approached $1 \mathrm{~ms}$, and a high pseudo fill factor and open circuit voltage have been obtained from a Suns Voc measurement. We conclude that these a-Si:H(p) layers are very promising for the application in high performance silicon heterojunction solar cells without using an intermediate a-Si:H(i) layer.
\end{abstract}

\section{INTRODUCTION}

The silicon heterojunction solar cell (SHJ), with a record efficiency of $22.3 \%$ reported by Sanyo in 2007 [ 1 ], has attracted extensive attention all over the world. Recently $23 \%$ have been reached (http://www.pvtech.org/news/_a/sanyo_a_hit_with_23_solar_cell_ efficiency_record/)

So far, many efforts have been made in order to understand the role of the amorphous Si layer in the heterojunction solar cell such as: 1) modeling the surface passivation of the amorphous silicon layer on the crystalline silicon wafer to understand its fundamental physical mechanism [ 2 ]; 2) studying and engineering the electronic band structure in order to improve the charge transport across the silicon heterojunction structure [3];3) experimentally optimizing the amorphous silicon deposition condition aiming at a high quality layer and device $[4,5]$.

The SHJ process is already well-known for its outstanding properties compared to the conventional solar cell process: 1) the SHJ formation is a process carried out at low temperature $\left(<200^{\circ} \mathrm{C}\right)$ with a very short deposition period; 2) doped a-Si:H for emitter and back surface field (BSF) can be produced via tuning the dopant species and deposition parameters; 3) a-
$\mathrm{Si}: \mathrm{H}(\mathrm{i})$ was demonstrated to result in excellent surface passivation [6].

High quality a-Si:H(i) thin layers (thickness of around $5 \mathrm{~nm}$ ) play a very important role in the performance of the usual SHJ cells. To achieve such extremely thin and homogenous layers with a high surface passivation is not an easy task and the characterization of such kind of layers ex-situ is almost impossible due to their instability in ambient. Olibet et al. found that the surface passivation of a-Si:H(i) varied with the layer thickness and the best surface passivation was obtained at a layer thickness of $40 \mathrm{~nm}$. The passivation degraded both with decreasing and increasing thickness, due to either less layer relaxation or mechanical stress respectively [7]. Therefore, for passivation, the intermediate a$\mathrm{Si}: \mathrm{H}(\mathrm{i})$ layer with a thickness of $5 \mathrm{~nm}$ is not the best choice. However, with increasing layer thickness, such insulating layers can greatly reduce the charge transport between silicon wafer and amorphous doped layer, and also increase the light absorption.

Depositing a-Si:H(p/n) directly on the Si wafer is another option but there is usually an inferior surface passivation due to the recombination via the interface defects, which are produced by the dopant species. So far, there was only one report of succeeding in the high performance Si heterojunction cell produced without intermediate a-Si:H(i) from K. V. Maydell et. al.[8].

In this work, we apply a new method, remote linear radio frequency plasma-enhanced CVD (rfPECVD), to produce high quality a-Si:H(p) layers directly on the mono-crystalline silicon (100) wafer. It not only results in a very good surface passivation that is comparable to its intrinsic counterpart, but the layers also posses a high dark conductivity. The surface passivation of such a$\mathrm{Si}: \mathrm{H}(\mathrm{p})$ as a function of layer thickness and applied dopant flow rate is investigated. The lowest surface recombination velocity $S_{\text {eff }} \sim 7 \mathrm{~cm} / \mathrm{s}$ is obtained at a layer thickness of $\sim 70 \mathrm{~nm}$. A range of dopant gas flow rates for achieving both good surface passivation and conductivity has been defined. A stable effective lifetime $\left(\tau_{\text {eff }}\right)$ is demonstrated for a thin ( $20 \mathrm{~nm})$ a-Si:H(p) layer. The surface passivation of various cell structures is investigated and the pseudo fill factor and open circuit voltage $\left(\mathrm{V}_{\mathrm{oc}}\right)$ are studied by using the Suns $V o c$ measurement.

\section{EXPERIMENTS}


N-type float zone silicon (100) wafers (diam. $=100 \mathrm{~mm}$ ) with a thickness of $250 \mu \mathrm{m}$ and a resistivity of $2.3 \Omega \mathrm{cm}$ were used as the substrates for the amorphous silicon layer deposition. Prior to the deposition, chemical cleaning and a short HF dip were performed. After the HF dip, the wafer is transported into the high vacuum chamber of the rf-PECVD within a short time to avoid surface degradation.

For studying the single a-Si:H(p) layer, we deposit a thick, passivating a-SiN $\mathrm{N}_{\mathrm{x}}$ layer [9] on one side of the wafer by using a separate Microwave PECVD system. This assures a good surface passivation, which normally yields $\tau_{\text {eff }}>1 \mathrm{~ms}$. The a-Si:H(p) is deposited on the other side for this study.

The a-Si:H(p) layer deposition is carried out in the p-chamber of rf-PECVD system equipped with a rf plasma source (13.56 Mhz) [10]. The PECVD chamber has a layout as shown in figure 1. This system is able to deposit layers with mild ion energy, which would not easily damage the wafer surface. Beside $\mathrm{SiH}_{4}$ and $\mathrm{H}_{2}$ gas, a $\left(\mathrm{B}_{2} \mathrm{H}_{6}\right.$ $+\mathrm{H}_{2}$ ) gas mixture was introduced in the deposition for the p-type doping process. The ratio B:Si was varied by tuning the gas flow rate.

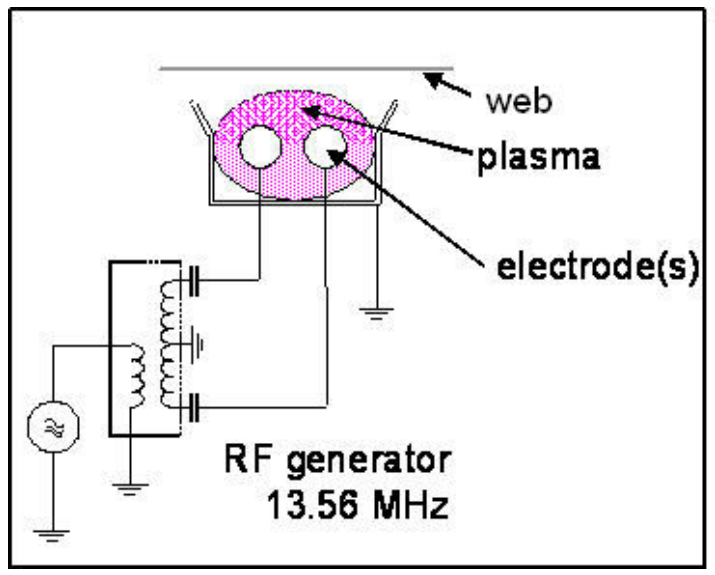

Figure 1: schematic drawing of the remote linear radio frequency plasma enhanced CVD system. The web carries the wafers (face down).

In the formation of the cell structure of a$\mathrm{Si}: \mathrm{H}(\mathrm{p}) / \mathrm{c}-\mathrm{Si}(\mathrm{n}) / \mathrm{a}-\mathrm{Si}: \mathrm{H}(\mathrm{n}), \quad \mathrm{a}-\mathrm{Si}: \mathrm{H}(\mathrm{n})$ layers were deposited in the n-chamber of rf-PECVD system with the same plasma source. $\left(\mathrm{PH}_{3}+\mathrm{H}_{2}\right)$ gas mixture was used as dopant gas for the n-type doping.

Post annealing was commonly applied right after the deposition in our experiments. It was performed in a conventional box furnace in air. The $\tau_{\text {eff }}$ was measured by the Sinton WT 120 in the transient mode and it was extracted at a charge carrier density of $10^{15} / \mathrm{cm}^{3}$. The stability of surface passivation as a function of time was measured for a thin a-Si:H(p) layer ( 20nm), which was kept in the dark and in the air. ex-situ ellipsometry (SE850, Sentech Instrument Gmbh) was used to determine the amorphous nature of the deposited layers. The dark conductivity measurement was carried out by the two probe method using a Keithley 595 Quasistatic CV Meter. The conductivity samples were simultaneously deposited on glass substrates; coplanar aluminum contacts were later evaporated by using the ebeam. To quantify the $\mathrm{SHJ} \mathrm{p} / \mathrm{n} / \mathrm{n}$ cell structures, ITO layers were sputtered on both front and rear sides of wafer and a Ag layer was sputtered on the rear side as the back contact. SunsVoc measurements resulted in the pseudo fill factor and open circuit voltage $\left(\mathrm{V}_{\mathrm{oc}}\right)$.

\section{RESULTS AND DISCUSSIONS}

Figure 2 plots the surface recombination velocity $\mathrm{s}$ of the a-Si:H(p) layers on $\mathrm{Si}(100)$ as a function of layer thickness. As shown in the inset of figure 2, the samples include a-Si:H(p) layer deposited on the front side of wafer and a high
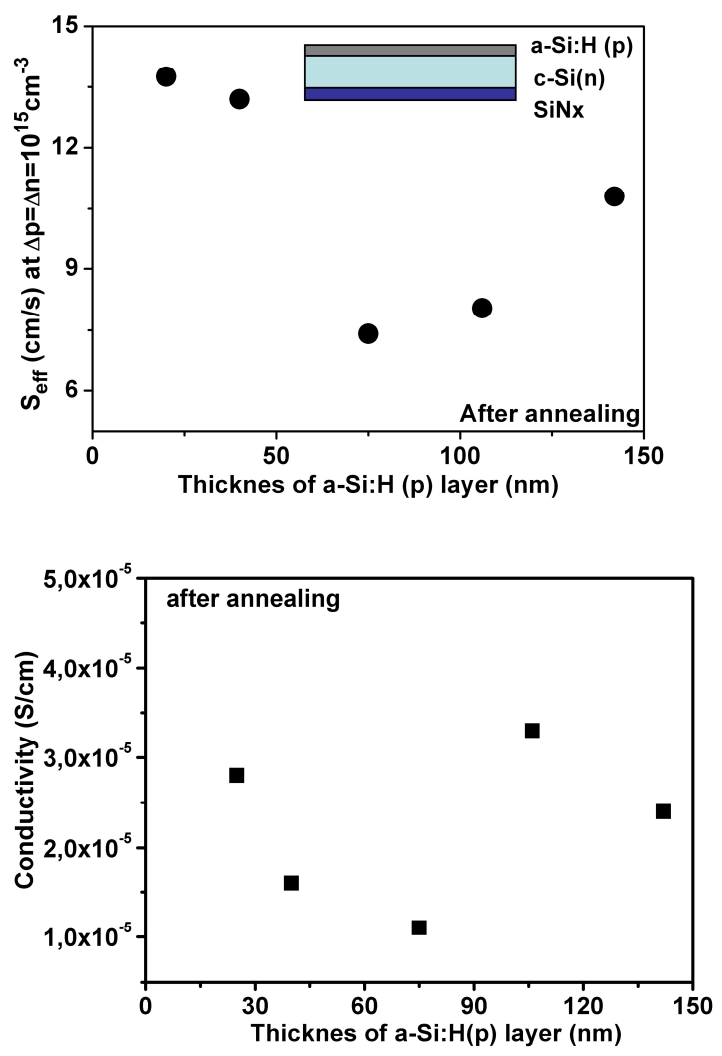

Figure 2: surface recombination velocity of annealed a-Si:H(p) layers as function of layer thickness (upper); dark conductivity obtained for the same a-Si:H(p) layers as function of layer thickness (lower). 
quality SiNx layer deposited on the rear side of wafer, which can secure a very good surface passivation. The recombination velocity is converted from measured $\tau_{\text {eff }}$ and is approximated by the equation $\frac{1}{\tau_{\text {eff }}}=\frac{1}{\tau_{\text {bulk }}}+\frac{2 S}{W}$, in which $\tau_{\text {bulk }}$ is the bulk lifetime assumed to be infinite; $S$ is the recombination velocity and $W$ is the wafer thickness. $S$ is thus practically the average of the respective $\mathrm{S}$ of the $\mathrm{SiNx}$ and the $a-\operatorname{Si}(\mathrm{p}): \mathrm{H}$. It is noticed that the smallest recombination velocity is obtained for the layer with a thickness of $\sim 70 \mathrm{~nm}$. Furthermore, it shows a comparable surface passivation to that of the a-Si:H(i) layer reported by Olibet et al. [11]. When reducing the layer thickness down to $20 \mathrm{~nm}$, the recombination velocity increases to 2 times higher than that at 70 $\mathrm{nm}$ indicating the degradation of the surface passivation for thinner a-Si:H(p) layer. This shows a similar trend as the behavior of a-Si:H(i) and it is attributed to the insufficient relaxation of the thin layer [7]. In the lower part of figure 2, the dark conductivity has been extracted by using two probe measurements. Clearly, all the results are well above $1 \times 10^{-5} \mathrm{~S} / \mathrm{cm}$ strongly demonstrating that the deposited layers are effectively doped.

To investigate the structure of the deposited layer, we applied an ex-situ ellipsometry measurement. As shown in figure 3, layers with high $\tau_{\text {eff }}$ show the typical curve for amorphous $\mathrm{Si}$. However, for the one with very low $\tau_{\text {eff }}=35 \mu \mathrm{s}$, as shown by the blue dashed curve, a crystalline-like step is found. These results are well consistent with previous ellipsometry studies $[12,13]$.

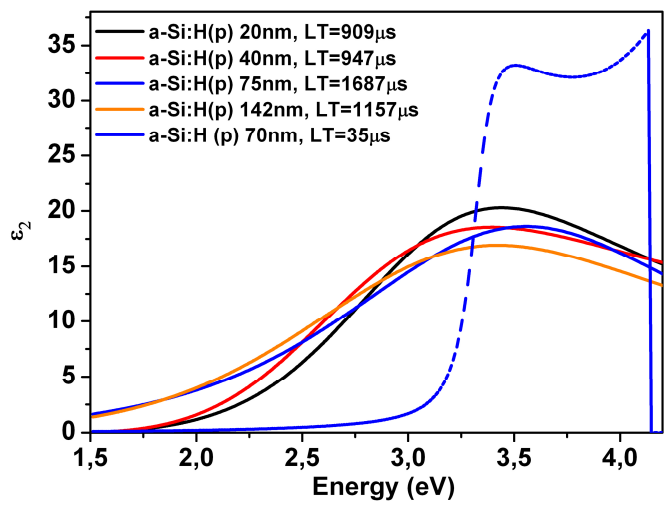

Figure 3: typical amorphous layer property is demonstrated from the ellipsometry measurement of the dielectric constant (imaginary part) as a function of wavelength.

Stability of surface passivation as function of time is another important issue because it can strongly impact to the performance of heterojunction solar cells. In this study, the surface passivation stability was investigated by means of measuring the $\tau_{\text {eff }}$ for an annealed a-Si:H(p) layer with a thickness of $\sim 20 \mathrm{~nm}$, which does not have any protecting capping layer and is stored in the dark, and in air. $\tau_{\text {eff }}$ was measured after every 10 days as shown in figure 4 . Only slight fluctuations of the measured lifetime of less than $5 \%$ are noticed. There is no obvious degradation of surface passivation for the deposited thin a-Si:H(p) layer even for more than a month after the initial annealing. So far, we don't have the results concerning to the passivation stability of such kind of $p$-layer storing under the light yet.

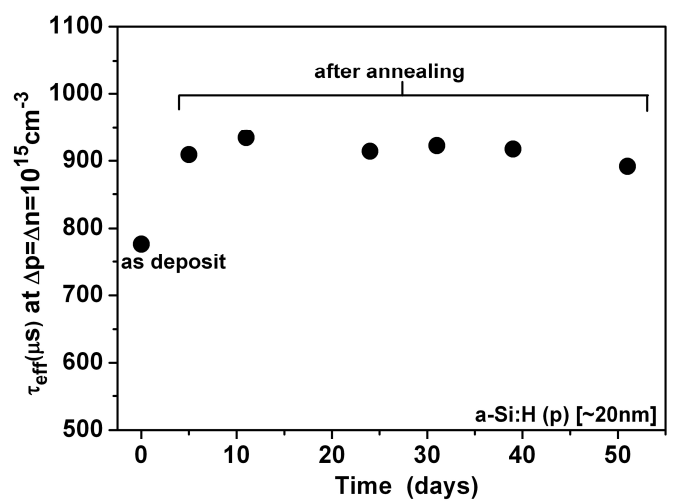

Figure 4 investigation of lifetime stability of deposited a-Si:H(p).

The influence of the dopant flow rate in the deposition on the surface passivation was also studied. The surface recombination velocity and dark conductivity after the annealing process, plotted as a function of dopant gas flow rate, are indicated in figure 5 . The surface recombination velocity and dark conductivity both decrease with reduced flow rate of dopant gas. These results qualitatively suggest that reducing the dopant concentration in the deposited layer can suppress

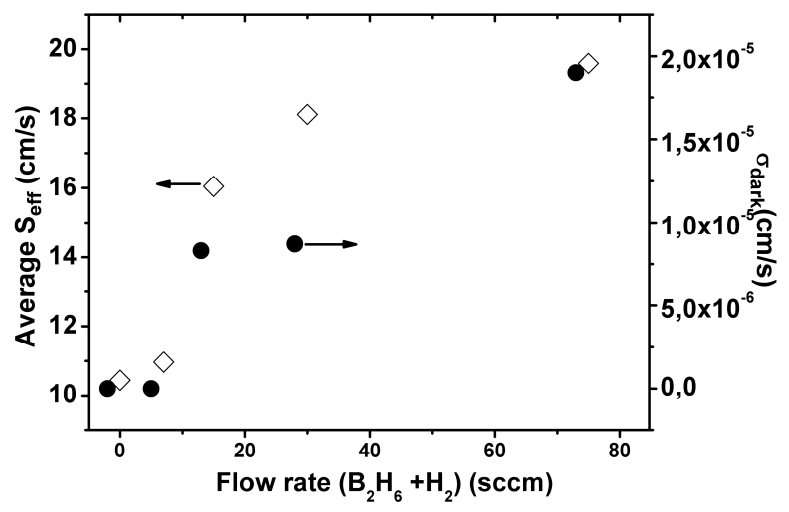

Figure 5 surface recombination velocity and dark conductivity of a-Si: $\mathrm{H}(\mathrm{p})$ [thickness $=50 \mathrm{~nm}$ ] are indicated as function of applied dopant gas flow rate. 
the density of defects which induce charge recombination at the interface. However, with a too low flow rate (i.e. $<15 \mathrm{sccm}$ ), although the surface passivation is enhanced, there is a large decrease of the dark conductivity of deposited a-Si:H(p). Based on these results, we suggest that: 1) with very low flow rate in terms of very low dopant concentration, a higher quality amorphous layer, which is comparable to the a-Si:H(i), can be achieved. This is possibly assisted by the so called "microdoping", which may cause the field effect passivation at the interface [2]; 2) to achieve a reliable surface passivation and good conductivity simultaneously for the deposited a-Si:H(p) layer, a compromise doping process should be considered.

High quality doped a-Si:H(p) layers with and without a-Si:H(i) buffer layers were deposited as an emitter on the polished front surfaces of $n$-type

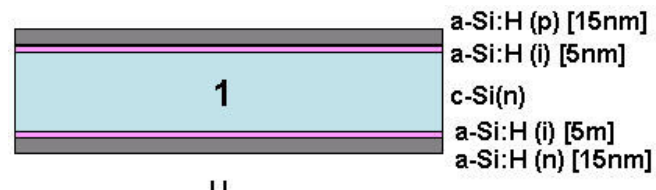

$\Downarrow$
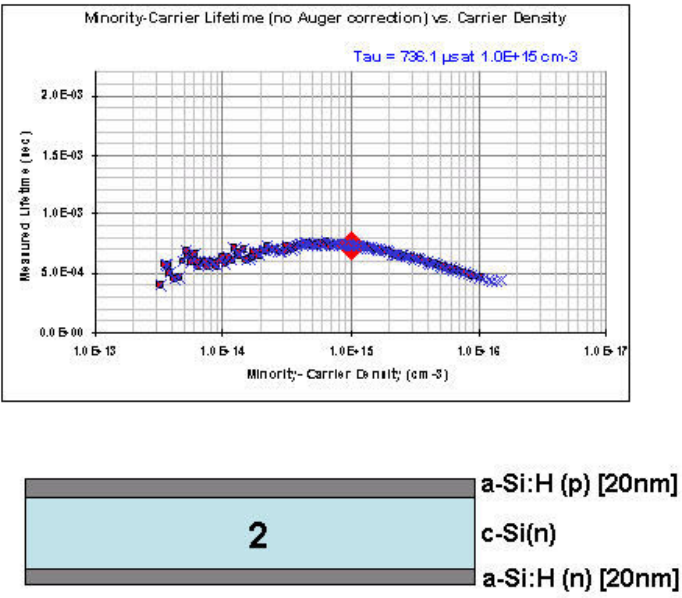

$\Downarrow$

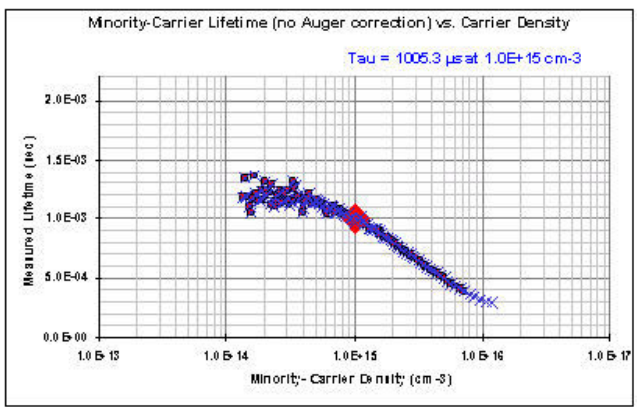

Figure 6 schematic drawing of stack layer 1 and corresponding minority charge carrier lifetime (upper); schematic drawing of stack layer 2 and corresponding minority charge carrier lifetime (lower). float zone $\mathrm{Si}(100)$ wafer as shown in the schematic drawings of figure 6 . An a-Si:H(n) layer was deposited on the rear side of wafer as BSF, also with and without intrinsic buffer layer. Stack 1 is formed by a-Si:H(p) /c-Si(n) /a-Si:H(n) and stack 2 is formed by a-Si:H(p) /a-Si(i) /c-Si(n) /a-Si:H(i) /a$\mathrm{Si}: \mathrm{H}(\mathrm{n})$. The a-Si:H(i) layers in stack 2 were introduced to improve the passivation and produced separately in either $\mathrm{n}$ or $\mathrm{p}$ chamber; the doped layers were deposited on top without breaking the chamber vacuum. The surface passivation quality of each stack is indicated accordingly in figure 6 . A better surface passivation ( $\tau_{\text {eff }}=1.0 \mathrm{~ms}$ ) is observed for stack 2 than for stack $1\left(\tau_{\text {eff }}=0.74 \mathrm{~ms}\right)$. The surface passivation achieved for stack 2 is comparable to the single a$\mathrm{Si}: \mathrm{H}(\mathrm{p})$ shown in figure 4 indicating a consistent layer quality. The lower surface passivation for stack 1 is apparently related to the very thin $(\sim 5$ $\mathrm{nm})$ a-Si:H(i) layer.

In table 1, we compare the dark conductivity measured for single a-Si:H(p) layers with the a-Si:H(p)/a-Si:H(i) stack. After annealing, the conductivity is increased for both cases, but the conductivity of the i/p stack is about $2-3$ orders of magnitudes lower than those of the single a$\mathrm{Si}: \mathrm{H}(\mathrm{p})$ layers. This indicates that the conductivity can be greatly improved by removing the intermediate a-Si:H(i) layer. Whilst still keeping a good surface passivation, such kind of a-Si:H(p) layer will be favored for the device fabrication.

\begin{tabular}{|c|c|c|}
\hline & $\begin{array}{c}\sigma_{\text {Dark }}(\mathrm{S} / \mathrm{cm}) \\
\text { before } \\
\text { anneal }\end{array}$ & $\begin{array}{c}\sigma_{\text {Dark }}(\mathrm{S} / \mathrm{cm}) \\
\text { after anneal }\end{array}$ \\
\hline p-layer 1 [20nm] & $2.0 \mathrm{E}-7$ & $8.3 \mathrm{E}-6$ \\
\hline p-layer 2 [20nm] & $7.2 \mathrm{E}-7$ & $3.7 \mathrm{E}-5$ \\
\hline $\mathrm{i}[5 \mathrm{~nm}] / \mathrm{p}[15 \mathrm{~nm}]$ & $1.0 \mathrm{E}-9$ & $6.8 \mathrm{E}-8$ \\
\hline
\end{tabular}

Table 1 dark conductivity measured on a-Si:H(p) layer and on a-Si:H(p) / a-Si:H (i) stack,

By using the SunsVoc measurement, we further demonstrate the advantages of depositing the a-Si:H(p) layer directly on the Si wafer. In figure 7 , pseudo J-V curves show the high quality silicon heterojunction without indicating any obvious shunt for both kinds of the stack layers. Pseudo fill factor and $V_{\text {oc }}$ are extracted by averaging measurements on 5 different points on the wafers. As seen in figure 7 , a high $V_{\text {oc }}(>670$ $\mathrm{mV}$ ) has been obtained for both stacks. It indicates that the surface passivation of both stack layers is still good after ITO deposition and Ag back contact deposition. A much higher pseudo fill factor FF = 0.84 is obtained for stack 2 than that (pseudo FF = 0.80 ) of stack 1 . These results indicate that the stack layer without intermediate a-Si:H(i) has a better behavior than the one with the a-Si:H(i) when neglecting the series resistance. Hence, we 


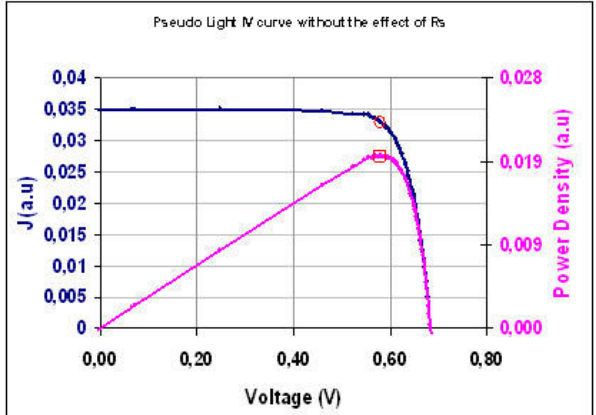

ITO/a-Si:H(p)/a-Si:H(i)/c-Si(n)/a-Si:H(i)/a-Si:H(n)/ITO/Ag PseudoFf $=0.8$

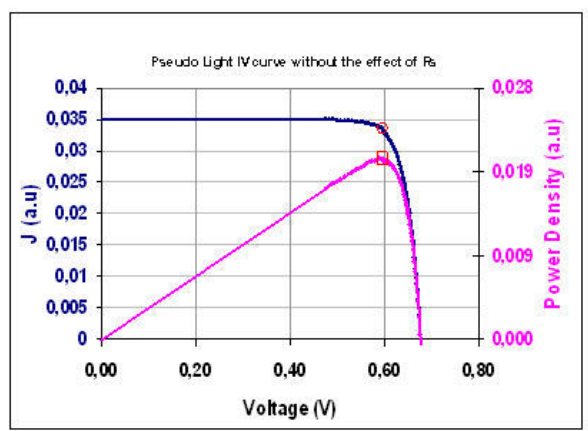

ITO/a-Si:H(p)/c-Si(n)/a-Si:H(n)/TO/Ag Pseduo $\mathrm{FF}=\mathbf{0 . 8 4}$

Figure $7 \mathrm{~J}-\mathrm{V}$ curve is plotted for stack 1 (upper) \& 2 (lower) respectively and the extracted pseudo fill factor is shown.

believe, also in the real solar cell, a better performance will be achieved on the stack layer 2 since it has also been demonstrated to have a better conductivity in the doped layers, and contact resistance can be expected to be further reduced in the absence of intrinsic buffer layers [14].

\section{CONCLUSIONS}

High quality a-Si:H(p) layer has been successfully deposited directly on the n-type float zone $\mathrm{Si}(100)$ wafer using remote linear rf-PECVD. The thin a-Si:H(p) layer $(\sim 20 \mathrm{~nm})$ results in an effective surface passivation, which is comparable to that of the conventionally used a-Si:H (i) layer. It also shows stability of the good surface passivation with time, in the dark, and in air. Furthermore, the conductivity of the a-Si:H(p) layer has been greatly improved by annealing without losing the high surface passivation property. This indicates a potentially reliable application of the a$\mathrm{Si}: \mathrm{H}(\mathrm{p})$ layer for the silicon hereojunction solar cell resulting in a better conversion efficiency performance. Future studies will mainly be focused on front contact optimization and series resistance improvement.
We acknowledge the financial support by SenterNovem, for QCpassi, project no EOSLT 03011.

\section{REFERENCES}

1. S. Taira, Y. Yoshimine, T. Baba, M. Taguchi, T. Kinoshita, H. Sakata, E. Maruyama, M. Tanaka, 22nd, EPSEC, Milan, Italy, 3-7 September, 2007.

2. S. Olibet, E. Vallat-Sauvainm C. Balif, Phys. Rev. B, Vol 76, 035326,2007.

3. T.H. Wang, M.R. Page, E. Iwaniczko, D.H. Levi, Y. Yan, H.M. Branz and Q. Wang, $14^{\text {th }}$ workshop on crystalline silicon solar cells and module, Winter Park, Colorado, August 8-11, 2004.

4. T.H. Wang, E. Iwaniczko, M.R. Page, D.H.Levi, Y. Yan, H.M. Branz, Q. Wang, Thin Solid Film, Vol. $501,284,2006$.

5. H. Fujiwara and M. Kondo, Appl. Phys. Lett. Vol. 90, 013503, 2007.

6. I. J. Pankove and M. L. Tarng, Appl. Phys. Lett. Vol. 34, 156, 1979

7. S. Olibet, $\mathrm{PhD}$ thesis "Properties of interface in amorphous / crystalline silicon heterojunction", Page 117, 2009.

8. K.V.Maydell, E. Conrad and M.Schmidt, Prog. in Photovolt: Res. Appl. Vol 14, 289, 2006.

9. W. Soppe, H. Rieffe, A. Weeber, Prog. in Photovolt: Res. Appl. Vol.13, 551, 2005.

10. H. Schlemm, M. Fritzsche, D. Roth, Surf \& Coat. Tech., Vol. 200, 958, 2005.

11. S. Olibet, E. vallat-Sauvain and C. Ballif, $21^{\text {st }}$, European Photovoltaic Conference, Dresden, Germany, September 2006.

12. D. Levi, E. Iwaniczko, M. Page, Q. Wang, H. Branz and T. Wang, IEEE 4th world conference on Photovoltaic Energy Conversion, May 7-12, 2006.

13. H. Fujiwara and M. Kondo, Appl. Phys. Lett. Vol. 86, 032112, 2005

14. S. Olibet, $\mathrm{PhD}$ thesis "Properties of interface in amorphous / crystalline silicon heterojunction", Page 32-35, 2009.

\section{ACKNOWLEDGEMENTS}

